\section{Secretory Expression of Rehmannia Class III Chitinase in Bacillus subtilis}

\author{
Cheol-Ho Pan ${ }^{1, *}$ and Su-Il Kim² \\ ${ }^{1}$ Natural Product Research Center, Korea Institute of \\ Science and Technology Gangneung Institute, \\ Gangneung 210-340, Korea \\ ${ }^{2}$ Department of Agricultural Biotechnology, College of \\ Agriculture and Life Sciences, Seoul National \\ University, Seoul 151-921, Korea
}

Received March 10, 2008; Accepted April 17, 2008

Key words: Bacillus subtilis, class III chitinase, recombinant protein, Rehmannia glutinosa

Chitinases (EC 3.2.1.14) are defined as enzymes cleaving a bond between the $\mathrm{C} 1$ and $\mathrm{C} 4$ of two consecutive $\mathrm{N}$-actyl-D-glucosamines (GlcNAc) of chitin and have received much attention because of their wide range of applications such as protection of plants against the fungal pathogen [Sundheim et al., 1988] and particularly the production of chitooligosaccharides [Usui et al., 1990] due to their broad applications in medicine, agriculture, cosmetics, and food [Boller, 1986; Gooday, 1986; Ordentlich et al., 1988].

The JHIII gene (NCBI accession AY206666) encoding an acidic class III chitinase was isolated from the leaf of Rehmannia glutinosa (Korean name: ji-huang), which is commonly used in the oriental medicine [Kim and Pan, 2007]. To investigate the enzymatic properties of JHIII protein, the corresponding gene, JHIII, was first expressed in Escherichia coli. Although E. coli has been the most commonly employed in the production of heterologous proteins and many heterologous proteins have been expressed directly or as fusion proteins in the cytoplasm of E. coli, the recombinant JHIII (rJHIII) was in an extremely insoluble form that it showed little chitinolytic activity (data not shown). rJHIII was purified by immobilized metal affinity chromatography using a

\footnotetext{
*Corresponding author

Phone: +82-33-650-7115; Fax: +82-33-650-7299

E-mail: panc@kist.re.kr
}

doi:10.3839/jabc.2008.015 nickel agarose resin (His-bind, Merck, Germany) as a packing material under the denaturing conditions and used as an immunogen for the production of an anti$\mathrm{rJHIII}$ antibody in the Balb b/c mouse. Other expression systems, such as the Bacillus expression system, were also explored to obtain the functional rJHIII. The greatest advantage of $B$. subtilis is its ability to secrete proteins directly into the culture medium. A further advantage of this direct secretion is that the proteins are often correctly folded, disulfide-bonded, and soluble.

pUB140, a plasmid vector for the Bacillus expression, was used [Son et al., 2001]. However, in the present study, for the sake of convenient cloning, a shuttle vector was made between E. coli and B. subtilis. An origin of replication (ori) and a selection marker (amp) from pUC18 were introduced into pUB140, resulting in a shuttle vector pUBL140, which can be replicated in both $B$. subtilis and E. coli (Fig. 1A). For the purpose of overexpression and secretion of a foreign protein in $B$. subtilis, a promotor (amyR2) and a signal sequence (SS) were isolated from $B$. subtilis NA64, an $\alpha$-amylasehyperproducing strain, [Yamazaki et al., 1983] and cloned into the p8A-1 plasmid. They were then inserted into pUBL140, resulting in pUBLamy (Fig. 1B). The promotor amyR2 is known to be transcribed at an early stationary phase of the cell growth. An expression vector, pUBLJHIII, was constructed to express $J H I I I$ gene in $B$. subtilis. The putative mature protein region of JHIII gene was amplified by PCR from the genomic DNA using the synthetic primers, JHB (5'-GGATCCAGGCAAAATCT CAATCTACTGG-3') and JHP (5'-CTGCAGTCAAACA TCCTTCTTGATAGAAG-3'). The PCR product was cloned into pGEM-T Easy (Promega, Madison, WI) and sequenced. The JHIII gene fragment digested with BamHI and PstI was purified and inserted into the expression vector pUBLamy, which has been digested with the same restriction enzymes (Fig. 1B). The expression vector pUBLJHIII and the control vector pUBLamy were transformed into B. subtilis WB600 deficient in six extracellular proteases [Wu et al., 1991] according to the method described by Sadaie and Kada [1983] with a slight modification.

To express the recombinant enzymes, $B$. subtilis was inoculated into $50 \mathrm{~mL}$ LB media containing $10 \mu \mathrm{g} / \mathrm{mL}$ kanamycin and grown at $37^{\circ} \mathrm{C}$ for $16 \mathrm{~h}$ with shaking at $300 \mathrm{rpm}$. All overnight cultures were inoculated into the jar fermentor (MK250, Korea Fermentor Co., Incheon, Korea) containing 2 L LB media without antibiotics. The fermentor operating conditions were: $37^{\circ} \mathrm{C}$ with shaking at $500 \mathrm{rpm}$, and $3 \mathrm{kgf} / \mathrm{cm}^{2}$. The cell growth and the 

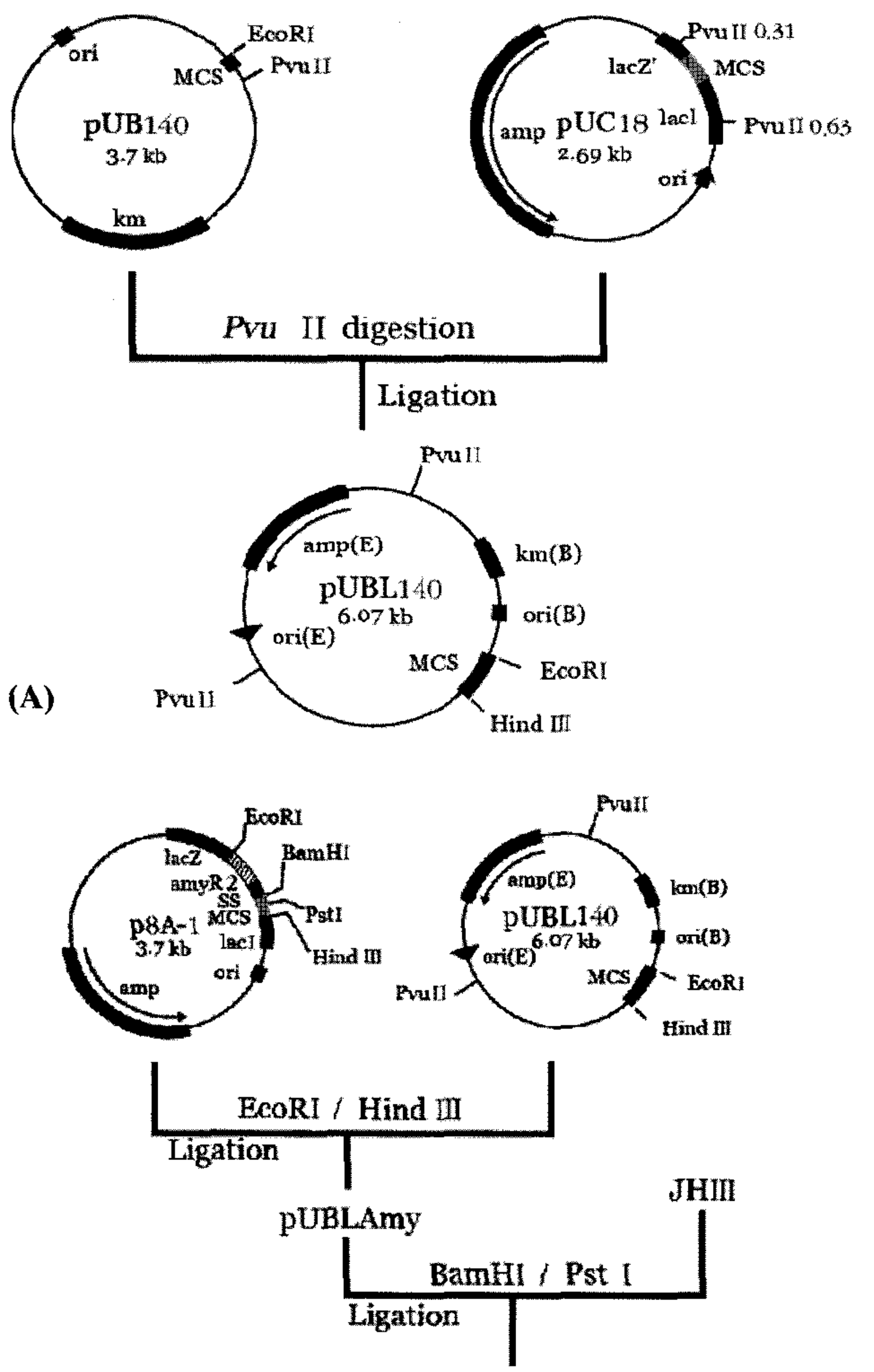

(B)

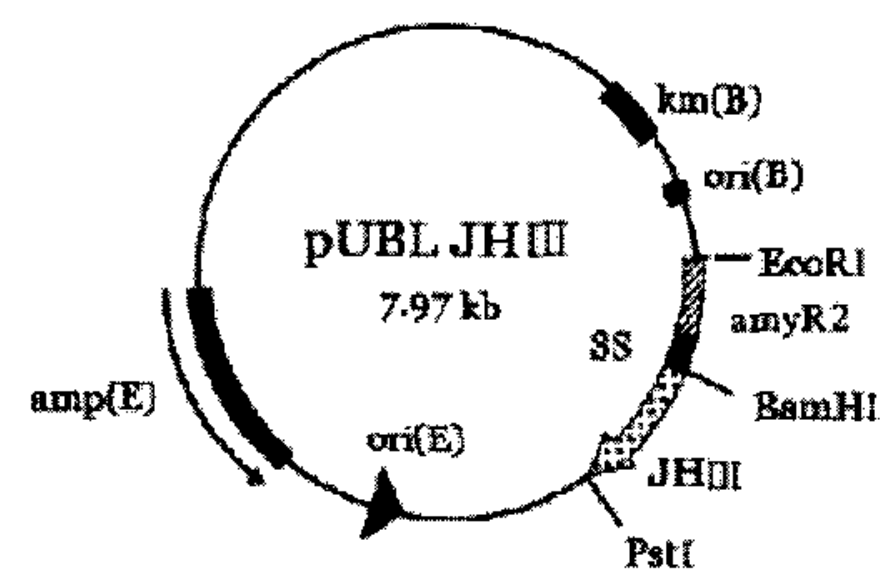

Fig. 1. Constructions of shuttle vector pUBL140 and expression plasmid pUBLJHIII. (A) A $2.35-\mathrm{kb}$ PvuII fragment from $\mathrm{pUC} 18$ containing the origin of replication (ori) and the selection marker (amp) for E. coli was inserted into the PvuII site of the Bacillus vector pUB140. MCS indicates a multicloning site. (B) Approximately 1 $\mathrm{kb}$ EcoRI-HindIII fragment (amyR2-SS domain) of p8A-1 was inserted into pUBL140, resulting in pUBLamy. The ORF of JHIII gene was ligated into the expression vector pUBLamy. The amyR2 and the SS indicate the promotor and the signal sequence, respectively.

chitinase activity were measured every $2 \mathrm{~h}$ during the culture. The cell growth was monitored by measuring the absorbance at $600 \mathrm{~nm}$. The chitinase activity of the culture supernatant as well as that of the cell lysate were measured radiometrically with $\left[{ }^{3} \mathrm{H}\right]$ chitin as the substrate according to the Molano's method [Molano et al., 1977].
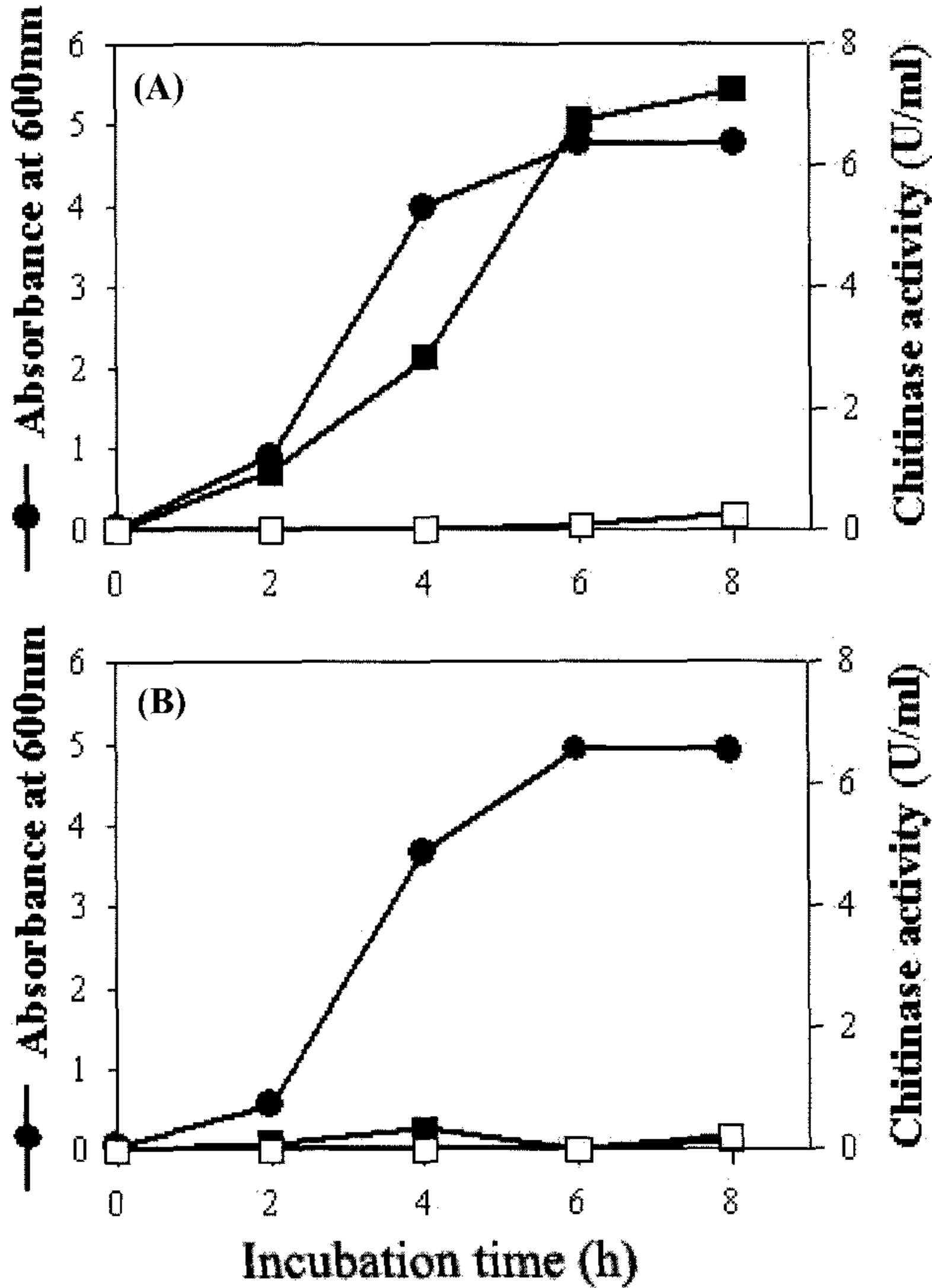

Fig. 2. Production of rJHIII by $B$. subtilis WB600 harboring pUBLJHIII and the cell growth in LB medium. B. subtilis WB600 cells containing pUBLJHIII (A) and pUBLamy (B) were cultured in the LB medium at $37^{\circ} \mathrm{C}$, and both their growth and enzyme activities of the culture supernatants (- $\square-$ ) and cell lysates (- $\square-$-) were measured every $2 \mathrm{~h}$.

One unit of the enzyme activity was defined as the amount of enzyme producing radioactivity corresponding to $1 \mathrm{nmol}$ of GlcNAc per minute under the given conditions. The protein content was determined by the bicinchoninic acid method [Smith et al., 1985] using BSA as a standard according to the manufacturer's instructions (Pierce Biotechnology, Rockford, IL). Both cultures began the exponential phase after $2 \mathrm{~h}$ of the lag phase and reached the stationary phase after $6 \mathrm{~h}$ incubation (Fig. 2). Only the chitinase activity of the culture supernatant of $B$. subtilis (pUBLJHIII) increased as the cells grew, and reached maximum in $8 \mathrm{~h}$ (Fig. 2A). This chitinase activity was due to the expression of JHIII gene, because no activity was detected in the culture of B. subtilis (pUBLamy) (Fig. 2B). In addition, no chitinase activity was detected within the cells of $B$. subtilis (pUBLJHIII), suggesting that the expressed protein was secreted efficiently into the medium (Fig. 2A). The yield of rJHIII from B. subtilis was $7.2 \mathrm{unit} / \mathrm{mL}$, and the specific activity of the culture broth was 3.4 unit/mg. The production of rJHIII was 


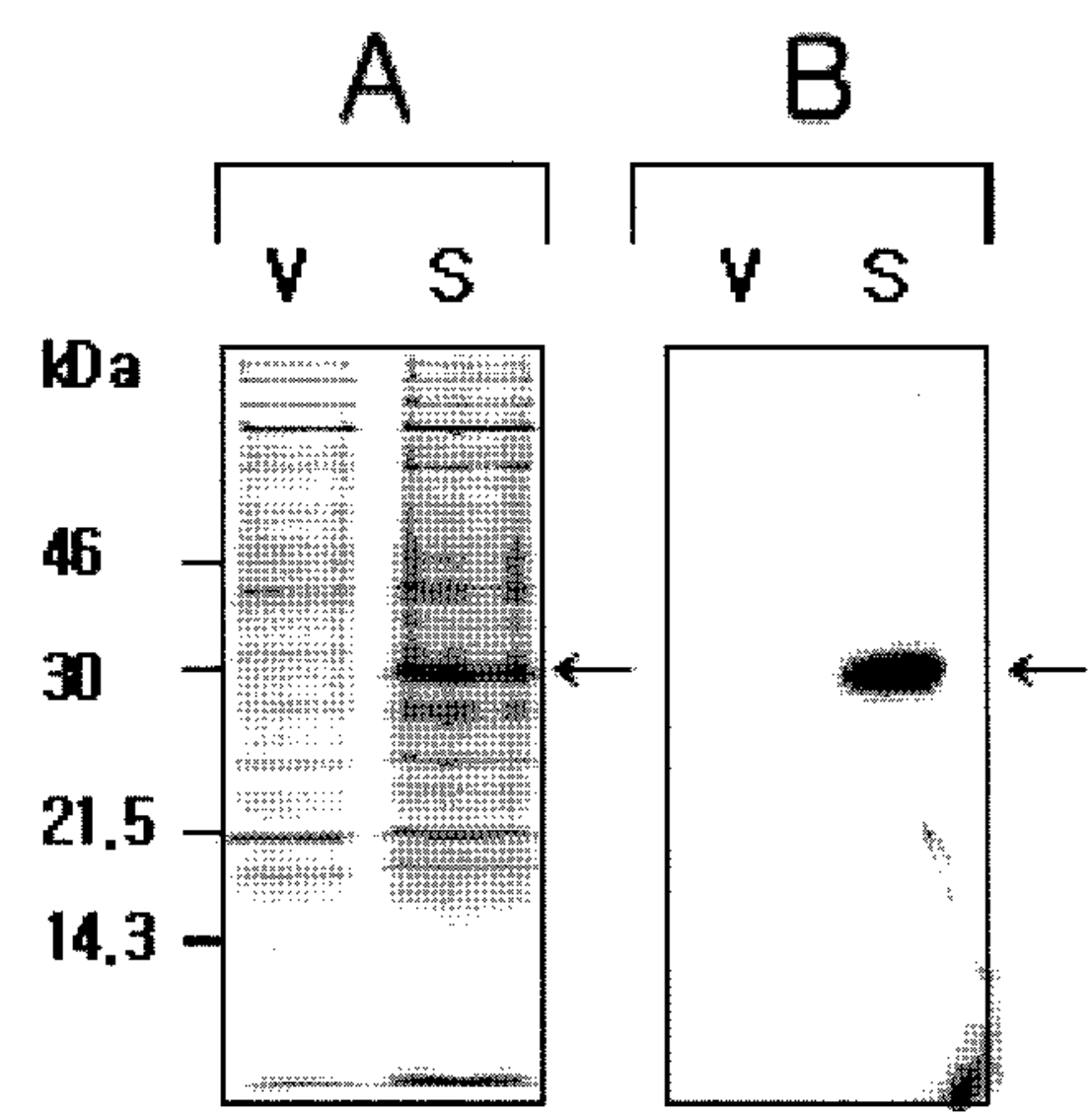

Fig. 3. SDS-PAGE (A) and immunoblot analysis (B) of rJHIII expressed in $\boldsymbol{B}$. subtilis. The expression of rJHIII was analyzed on $12.5 \%$ SDS-PAGE by Coomassie brilliant blue R-250 (A) as well as the anti-rJHIII antiserum (B). The molecular masses of the protein standards are given on the left side. The arrows indicate rJHIII. $\mathrm{V}$, the culture supernatant from $B$. subtilis WB600 transformed with pUBLamy; $S$, the culture supernatant from $B$. subtilis WB600 transformed with pUBLJHIII.

further confirmed by both SDS-PAGE and the immunoblot analysis (Fig. 3). The culture supernatants from $B$. subtilis WB600 with pUBLamy and pUBLJHIII after $8 \mathrm{~h}$ incubation were analyzed by $12.5 \%$ SDS-PAGE. The prominent band discovered from the culture supernatant of B. subtilis WB600 (pUBLJHIII) was estimated as 29 $\mathrm{kDa}$, which corresponded to the calculated molecular mass of rJHIII (Fig. 3A, lane S). The band was confirmed to be rJHIII expressed in B. subtilis WB600, because the anti-rJHIII antibody reacted only with the band (Fig. 3B, lane $S$ ). However, this band was not detected in the culture supernatant of B. subtilis WB600 (pUBLamy).

In conclusion, the plant chitinase JHIII was successfully expressed as an active and secretory form in B. subtilis. However, further study is necessary on the enzymatic characterization of rJHIII.

Acknowledgments. This work was supported by the grant (RTI05-01-02) from the Regional Technology Innovation Program of the Ministry of Commerce, Industry and Energy (MOCIE). Special thanks to professor Sui-Lam Wong of the University of Calgary for providing B. subtilis WB600.

\section{References}

Boller T (1986) Chitinase: A defense of higher plants against pathogens. In Chitin in Nature and Technology, Muzzarelli RA, Jeuniaux C, and Gooday GW pp. 223231, Plenum Press, New York.

Gooday GW (1986) Chitinase activities in mammals, fungi and bacteria. Ibid.

Kim SI and Pan CH (2007) Molecular cloning of acidic class III chitinase gene from Rehmannia glutinosa. $J$ Chitin Chitosan 12, 65-72.

Molano J, Duran A, and Cabib E (1977) A rapid and sensitive assay for chitinase using tritiated chitin. Anal Biochem 83, 648-656.

Ordentlich A, Elad Y, and Chet I (1988) The role of chitinase of Serratia marcescens in biocontrol of Sclerotium rolfsii. Phytopathology 78, 84-88.

Sadaie Y and Kata T (1983) Formation of competent Bacillus subtilis cells. J Bacteriol 153, 813-821.

Smith PK, Krohn RI, Hermanson GT, Mallia AK, Gartner FH, Provenzano MD, Fujimoto EK, Goeke BJ, Olson BJ, and Klenk DC (1985) Measurement of protein using bicinchoninic acid. Anal Biochem 150, 76-85.

Son JM, Pan CH, So MY, Chae YA, and Kim SI (2001) Growth inhibition of fungal pathogen by rice chitinase (CH16) expressed in Bacillus subtilis. J Chitin Chitosan 6, 41-44.

Sundheim L, Polawsky AR, and Ellingboe AH (1988) Molecular cloning of two chitinases genes from Serratia marcescens and their expression in Pseudomonas species. Physiol Mol Plant Pathol 33, 481-491.

Usui T, Matsui H, and Isobe K (1990) Enzymatic synthesis of useful chito-oligosaccharides utilizing transglycosylation by chitinolytic enzymes in a buffer containing ammonium sulfate. Carbohydr Res 203, 65-77.

Wu XC, Lee W, Tran L, and Wong SR (1991) Engineering a Bacillus subtilis expression-secretion system with a strain deficient in six extracellular proteases. $J$ Bacteriol 173, 4952-4958.

Yamazaki H, Ohmura K, Nakayama A, Takeichi Y, Otozai K, Yamasaki M, Tamura G, and Yamane K (1983) $\alpha$ Amylase genes (amyR2 and $a m y E^{+}$) from an $\alpha$-amylasehyperproducing Bacillus subtilis strain: molecular cloning and nucleotide sequences. $J$ Bacteriol 156, 327-337. 